\title{
Fluorescent microbead-based immunoassay for anti-Erysipelothrix rhusiopathiae antibody detection in cetaceans
}

\author{
Mar Melero ${ }^{1, *}$, Luis G. Giménez-Lirola ${ }^{2}$, Consuelo Rubio-Guerri ${ }^{1}$, \\ José Luis Crespo-Picazo ${ }^{3}$, Eva Sierra ${ }^{4}$, Daniel García-Párraga ${ }^{3}$, \\ Francisco Javier García-Peña ${ }^{5}$, Manuel Arbelo ${ }^{4}$, Teresa Álvaro ${ }^{3}$, Mónica Valls ${ }^{3}$, \\ José Manuel Sánchez-Vizcaíno ${ }^{1}$
}

${ }^{1}$ VISAVET Center and Animal Health Department, Veterinary School, Complutense University of Madrid, 28040 Madrid, Spain

${ }^{2}$ Department of Veterinary Diagnostic and Production Animal Medicine, College of Veterinary Medicine, Iowa State University, Ames, Iowa 50010, USA

${ }^{3}$ Veterinary Services, Oceanografic, Ciudad de las Artes y las Ciencias, 46013 Valencia, Spain

${ }^{4}$ Division of Histology and Animal Pathology, Institute for Animal Health, Veterinary School, University of Las Palmas de Gran Canaria, 35416 Canary Islands, Spain

${ }^{5}$ Bacteriology Department 2, Laboratorio Central de Veterinaria de Algete (MAGRAMA), 28110 Algete, Madrid, Spain

\begin{abstract}
A fluorescent microbead-based immunoassay (FMIA) for detection of antiErysipelothrix rhusiopathiae antibodies in pigs was adapted for use in cetaceans. The FMIA was validated and adjusted using serum samples from 10 vaccinated captive bottlenose dolphins Tursiops truncatus collected between 1 and 13 mo after immunization. The technique was then used to analyze specimens from 15 free-ranging cetaceans stranded alive on the Valencian Mediterranean coast between 2006 and 2014: 11 striped dolphins Stenella coeruleoalba, 3 Risso's dolphins Grampus griseus and 1 bottlenose dolphin Tursiops truncatus. One of these wild animals was confirmed to have died from E. rhusiopathiae septicemia, but no anti-E. rhusiopathiae antibodies were detected in its serum, pericardial fluid or milk samples. Another free-ranging individual, which lacked any signs or lesions that might be indicative of E. rhusiopathiae infection, showed high fluorescence intensity similar to that measured in captive dolphins at 6-13 mo after vaccination. These results suggest that this animal underwent an E. rhusiopathiae infection several months before stranding. The findings in the present study suggest that FMIA can be useful for detecting anti-E. rhusiopathiae antibodies in cetaceans, and its application to free-ranging animals is particularly interesting because of the great value of these specimens. Furthermore, the FMIA can be multiplexed to allow the determination of up to 100 analytes per sample in a single well, thereby reducing the cost, time and sample volume needed.
\end{abstract}

KEY WORDS: Erysipelothrix rhusiopathiae · Erysipelas · Antibodies $\cdot$ Fluorescent microbeadbased immunoassay · Cetaceans

\section{INTRODUCTION}

Erysipelothrix rhusiopathiae is a Gram-positive, non-acid-fast, rod-shaped bacterium that is a facultative anaerobe and does not form spores (Brooke \& Riley 1999, Wang et al. 2010). E. rhusiopathiae is ubiquitous and may persist in the environment,

\footnotetext{
${ }^{*}$ Corresponding author: mar.melero@ucm.es
}

including marine systems (Wang et al. 2010), for long periods in the presence of organic matter (Opriessnig \& Wood 2012). It has been isolated from the cutaneous slime of both fresh- and saltwater fish, insects, mollusks and crustaceans (Seibold \& Neal 1956, Lauckner 1985, Kinsel et al. 1997, Brooke \& Riley 1999, Wang et al. 2010, Opriessnig et al. 2013). 
E. rhusiopathiae is the causative agent of erysipelas, a disease that affects several species of mammals and birds, but mainly swine, turkeys (Kinsel et al. 1997, Dunn et al. 2001, Wang et al. 2010) and humans, in which E. rhusiopathiae infection is known as erysipeloid (Wang et al. 2010). In cetaceans, only the dermatologic and acute septicemic forms have been reported (Seibold \& Neal 1956, Chastel et al. 1975, Thurman et al. 1983, Buck \& Spotte 1986, Kinsel et al. 1997, Melero et al. 2011). Thrombosis of peripheral arteries and local tissue infarction cause pathognomonic rhomboid-shaped skin lesions (Sweeney \& Ridgway 1975).

The main source of infection in cetaceans is ingestion of contaminated fish (Geraci et al. 1966, Lauckner 1985, Suer \& Vedros 1988, Higgins 2000), but other sources can include humans with erysipeloid, contaminated food preparation sites, opportunistic colonization of wounds, and flying insects that serve as vectors of the pathogens between contaminated areas (Wood \& Shuman 1981). Therefore, most cases of erysipelas in cetaceans have been reported to occur in captive animals (Seibold \& Neal 1956, Geraci et al. 1966, Medway \& Schryver 1973, Thurman et al. 1983, Buck \& Spotte 1986, Bossart \& Eimstad 1988, Kinsel et al. 1997, Dunn et al. 2001), with infections rarely reported in free-ranging cetaceans (Chastel et al. 1975, Melero et al. 2011).

Determination of antibodies against E. rhusiopathiae in captive cetaceans allows for the assessment of vaccine efficiency (Lacave et al. 2001, Sitt et al. 2010, Bernal-Guadarrama et al. 2014). Its determination in unvaccinated captive and in free-ranging cetaceans may also allow detection of previous exposure and may assist in diagnosis. Main surface protective antigen (Spa) proteins have been described as suitable antigens for antibody detection against $E$. rhusiopathiae in pigs (Imada et al. 2003, To \& Nagai 2007, Giménez-Lirola et al. 2012). Here, we adapted a fluorescent microbead-based immunoassay (FMIA) based on a Spa protein-derived recombinant polypeptide that was specifically designed for detection of anti-E. rhusiopathiae antibodies in cetaceans. The FMIA was validated using samples from vaccinated captive bottlenose dolphins. It was then used to detect anti-E. rhusiopathiae antibodies in samples from free-ranging cetaceans stranded alive in the Mediterranean Sea, including the first case of erysipelas reported in a free-ranging bottlenose dolphin (Melero et al. 2011).

The main advantage of the FMIA is the capacity to develop multiplex diagnostic assays (Elshal \& McCoy 2006) for simultaneous determination of up to 100 analytes in the same sample, thereby reducing the cost, time and sample volume needed. FMIA can be more sensitive than traditional immunoassays (van Gageldonk et al. 2008, Giménez-Lirola et al. 2012, Sánchez-Matamoros et al. 2015). Although FMIA, based on Luminex's xMAP technology, has been used successfully in veterinary disease research (Clavijo et al. 2006, Anderson et al. 2011, Wagner et al. 2011, Giménez-Lirola et al. 2012, ChristopherHennings et al. 2013, Sánchez-Matamoros et al. 2015), the present study is, to the best of our knowledge, the first application of FMIA to cetaceans.

\section{MATERIALS AND METHODS}

A recombinant polypeptide of 415 amino acids derived from the E. rhusiopathiae spaA gene (GenBank accession number AB019124.1) was selected and produced as an antigen for anti-E. rhusiopathiae antibody detection in cetaceans. This protein was previously described as a suitable antigen for antiE. rhusiopathiae antibody detection in pigs (Imada et al. 2003, Ingebritson et al. 2010, Giménez-Lirola et al. 2012). The spaA gene fragment was commercially produced (GenScript), and the recombinant Spa protein-derived polypeptide was expressed in Escherichia coli by cloning into a pETDuet-1 expression vector (Novagen ${ }^{\circledR}$ ). The resulting fusion protein was purified from a clarified extract of E. coli Rosetta BL21 (DE3) using a nickel-nitrilotriacetic acid chelate affinity chromatography kit (PrepEase® His-tagged protein purification kit, USB Corporation) according to the manufacturer's instructions. FMIA was performed as previously described in methods used for antibody detection in pigs (Giménez-Lirola et al. 2012). All incubations were carried out at room temperature $\left(20-25^{\circ} \mathrm{C}\right)$ in the dark and under constant vortexing at $500 \mathrm{rpm}$ (Fisher Scientific). During the coupling reaction, antigen was coupled to carboxylated fluorescent microbeads (25 $\mu \mathrm{g}$ antigen per 5 million beads; bead region 64) using a 2-step carbodiimide reaction as previously described for pigs (Staros et al. 1986).

For the FMIA, serum samples were diluted 1:25 in assay buffer (0.1 M phosphate-buffered saline [PBS], $10 \%$ goat serum [Gibco®, Life Technologies], $0.05 \%$ Tween-20, pH 7.2) and mixed with $50 \mu \mathrm{l}$ of the suspension of antigen-coupled beads into each well of a 96-well flat bottom microtiter plate (Bio-Plex Pro ${ }^{\mathrm{TM}}$, Bio-Rad Laboratories). Plates were incubated for 60 min and washed 3 times with 0.1 M PBS containing $0.05 \%$ Tween-20 (PBST). Subsequently, $50 \mu \mathrm{l}$ of biotinylated Protein A from Staphylococcus aureus 
(Sigma-Aldrich) diluted 1:100 in assay buffer was added to each well, and the plates were incubated for another $30 \mathrm{~min}$. After 3 washes with PBST, $50 \mu \mathrm{l}$ of streptavidin-R-phycoerythrin (Sigma-Aldrich), diluted to $2.5 \mu \mathrm{g} \mathrm{ml}^{-1}$ in assay buffer, was added to each well and the plates were incubated for $30 \mathrm{~min}$. After an additional wash step, the beads were resuspended in $100 \mu \mathrm{l}$ of assay buffer and the reporter fluorescence of the beads was determined using a dual-laser BioPlex® 200 instrument (Bio-Rad) and analyzed using Bio-Plex Manager 6.0 software (Bio-Rad).

Median fluorescence intensity (MFI) was estimated from at least 50 beads; samples were evaluated in triplicate and the result was taken as the average of the 3 measurements. To assay levels of non-specific binding, we included on each plate 3 'background wells' consisting of coupled microspheres incubated with serum diluent in the absence of sample. The MFI response was corrected by subtracting the background well signal (the mean of the 3 wells) from the signal obtained for each sample.

The Spa-based FMIA was validated for cetaceans with serum samples from 10 captive bottlenose dolphins Tursiops truncatus vaccinated with Erysipelothrixbased inactivated vaccines, originally designed for use in pigs. Animals were housed at Oceanografic (Valencia, Spain) where a 12-mo booster schedule is followed. Serum samples were collected at intervals of $1,2,6,12$, and 13 mo after annual revaccination (or after the primo-vaccination, in the case of 2 individuals). All dolphins were vaccinated with ER BAC PLUS (Pfizer), with the exception of 1 individual that was vaccinated with ERYVAC (Intervet UK). For negative controls, serum samples from 20 unvaccinated captive bottlenose dolphins were also analyzed. Additionally, serial decimal dilutions of positive samples were tested.

After validation with samples of vaccinated animals, the FMIA was used to analyze serum samples from 15 free-ranging cetaceans which live-stranded (and later died) on the Mediterranean coast of Valencia Community (Spain). These specimens came from 11 striped dolphins Stenella coeruleoalba, 3 Risso's dolphins Grampus griseus, and the first case of erysipelas reported in a free-ranging bottlenose dolphin T. truncatus, stranded in the Mediterranean Sea (Melero et al. 2011).

Necropsy, histopathology, virology and bacteriology analysis were performed on all 15 free-ranging cetaceans. However, descriptive case evaluation included only the bottlenose dolphin and one Risso's dolphin that tested positive for anti-E. rhusiopathiae antibodies. Necropsies were performed following the standard protocols of the European Cetacean Society (Kuiken \& García-Hartmann 1991). Three sets of samples were prepared: one set was preserved in transport medium for bacteriological assays, a second set was stored at $-20^{\circ} \mathrm{C}$ for virological assays, and the third was preserved in formalin for histopathology examination.

For bacteriological analysis, swabs were collected from the mouth, blowhole, liver, spleen, lung, kidney and several sections of intestine from 4 striped dolphins and 2 Risso's dolphins; swabs were also collected from the cardiac valves of one Risso's dolphin. Each swab was preserved in 1 cryotube with FBP medium (see Gorman \& Adley 2004) containing 0.5\% active charcoal (Sigma-Aldrich), and the tubes were frozen at $-20^{\circ} \mathrm{C}$ until analysis. Samples processed for aerobic culture were inoculated onto Columbia agar with $5 \%$ sheep blood ( $\mathrm{CBA}_{i}$ Oxoid) and MacConkey agar plates (Oxoid), and then incubated overnight at $35^{\circ} \mathrm{C}$. For anaerobic culture, swabs were inoculated onto CBA and tryptose sulphite cycloserine agar plates (Oxoid) and incubated overnight at $35^{\circ} \mathrm{C}$ in anaerobic jars using the AnaeroGen system (Oxoid). Intestinal swabs were further analyzed using protocols based on ISO 10272-1:2006 (ISO 2006a,b) to detect Campylobacter and protocols based on ISO 6579:2002/Amd 1:2007 (ISO 2007) to detect Salmonella. Finally, swabs collected from liver, spleen, lung, and kidney were inoculated into $5 \mathrm{ml}$ of brain heart infusion (BHI) broth medium (Difco Laboratories) containing $0.1 \%$ Tween $80,5 \%$ horse serum, $50 \mu \mathrm{g} \mathrm{ml}^{-1}$ gentamicin, $0.1 \%$ sodium azide, and $0.001 \%$ crystal violet. After incubation at $37^{\circ} \mathrm{C}$ for $24 \mathrm{~h}, 1$ loopful of broth culture was streaked onto BHI agar containing $0.1 \%$ Tween $80,50 \mathrm{\mu g} \mathrm{ml}^{-1}$ gentamicin, and $0.1 \%$ sodium azide. The agar plates were incubated at $37^{\circ} \mathrm{C}$ for $48 \mathrm{~h}$ in an atmosphere of $5-10 \% \mathrm{CO}_{2}$ and then examined for the presence of typical Erysipelothrix colonies (Hassanein et al. 2001). All isolates considered significant after culture were further characterized using standard diagnostic techniques, including the API 20E, API 20NE, and API 20STREP systems (BioMérieux) and PCR-based analysis of 16S rRNA gene sequences when necessary. Bacteriology analysis of specimens from the bottlenose dolphin was previously described in Melero et al. (2011).

Morbillivirus can have immunosuppressive effects (Domingo et al. 1992, Schulman et al. 1997, Heaney et al. 2002). In order to examine whether infection with this virus could affect anti-E. rhusiopathiae antibody production, total RNA was extracted from skin, brain, lung, kidney, tonsil, prescapular and pulmonar lymph node tissues of all 15 free-ranging cetaceans 
using the NucleoSpin RNA II Kit (Macherey-Nagel). Cetacean morbillivirus (CeMV) diagnosis was performed using a real time RT-PCR assay as previously described (Rubio-Guerri et al. 2013).

During necropsy, tissues of all major organs and lesions were collected and stored in a fixative solution of $10 \%$ neutral buffered formalin for histological analysis. Fixed tissue samples were trimmed, processed by routine methods, embedded in paraffin, sectioned to a thickness of $5 \mu \mathrm{m}$, and stained with hematoxylin and eosin for examination by light microscopy.

\section{RESULTS AND DISCUSSION}

The presence of anti-Erysipelothrix rhusiopathiae antibodies in serum samples from all vaccinated dolphins was confirmed by Spa-based FMIA (Table 1). MFI values varied between individuals and decreased in most animals (except for Dolphins 5, 8, and 10) over time after vaccination (Table 1). No differences in MFI were related to the time of sample collection (after primo-vaccination or annual booster) (Table 1). Dolphin 2 displayed high MFI values only at 1 and 2 mo after vaccination, and Dolphin 1 presented the highest MFI values of all evaluated animals (except for Dolphin 6 at 12 mo after vaccination). However, a larger number of primo-vaccinated animals should be evaluated to establish the relationship between MFI value and the number of previous immunizations.

MFI values in negative controls were between -97.25 and 117.75 (mean $=-54.89$ ). Serial decimal dilutions showed that most positive samples could be diluted 100 times before becoming negative, while serum samples with MFI values near 1000 could not be diluted (Fig. 1).

Among free-ranging cetaceans, only 1 individual Risso's dolphin Grampus griseus (i.e. $6.7 \%$ of dolphins sampled) showed positive levels of anti-E. rhusiopathiae antibodies by Spa-based FMIA (Table 2).

The bottlenose dolphin Tursiops truncatus that died from an E. rhusiopathiae septicemia (Melero et al. 2011) tested negative for anti-E. rhusiopathiae antibodies in its serum, pericardial fluid, and milk specimens. Rhomboid-shaped skin lesions, which are a pathognomonic sign of erysipelas in many species (Wang et al. 2010), were observed in this animal (Fig. 2). In addition, E. rhusiopathiae was successfully cultured and isolated from skin and brain samples. Blood analysis revealed leukocytosis (48600 leukocytes $\mu^{-1}$ ) characterized by a neutrophilia
Table 1. Median fluorescence intensity (MFI) of serum from 10 vaccinated bottlenose dolphins sampled at different times after annual revaccination. Dolphin 1 was vaccinated with ERYVAC (Intervet), and Dolphins 2-10 with ER BAC PLUS (Pfizer). For Dolphins 1 and 2, time after vaccination was counted after receiving the booster dose at $21 \mathrm{~d}$. MFI values in bold are lower than the MFI value obtained from the free-ranging Risso's dolphin with a positive MFI value (shown in Table 2)

\begin{tabular}{|lcrrrr|}
\hline \multirow{2}{*}{ ID } & \multicolumn{5}{c|}{ Months after vaccination } \\
& 1 & \multicolumn{1}{c|}{2} & \multicolumn{1}{c|}{6} & \multicolumn{1}{c|}{12} & 13 \\
\hline 1 & 9671.21 & 8373.75 & 8321.75 & 4356.50 & \\
2 & 4968.67 & 2398.75 & $\mathbf{1 1 3 . 7 5}$ & $\mathbf{3 6 . 0 0}$ & $\mathbf{- 6 4 . 0 0}$ \\
3 & & 6819.67 & 3078.00 & $\mathbf{3 1 3 . 5 0}$ & \\
4 & 4710.17 & $\mathbf{9 7 4 . 6 7}$ & $\mathbf{7 8 8 . 2 5}$ & $\mathbf{3 9 9 . 2 5}$ & \\
5 & 2471.50 & & 3079.17 & 2454.75 & \\
6 & 6827.17 & & 5389.00 & 4746.25 & \\
7 & & 5277.50 & 1830.00 & $\mathbf{7 8 0 . 0 0}$ & $\mathbf{3 8 7 . 7 5}$ \\
8 & & 3982.67 & 1934.00 & 2867.00 & \\
9 & 2979.50 & 3001.00 & 2339.50 & $\mathbf{1 4 5 7 . 3 3}$ & \\
10 & & $\mathbf{1 2 7 3 . 5 0}$ & $\mathbf{1 2 5 1 . 2 5}$ & $\mathbf{1 5 0 8 . 0 0}$ & \\
& & & & & \\
\hline
\end{tabular}

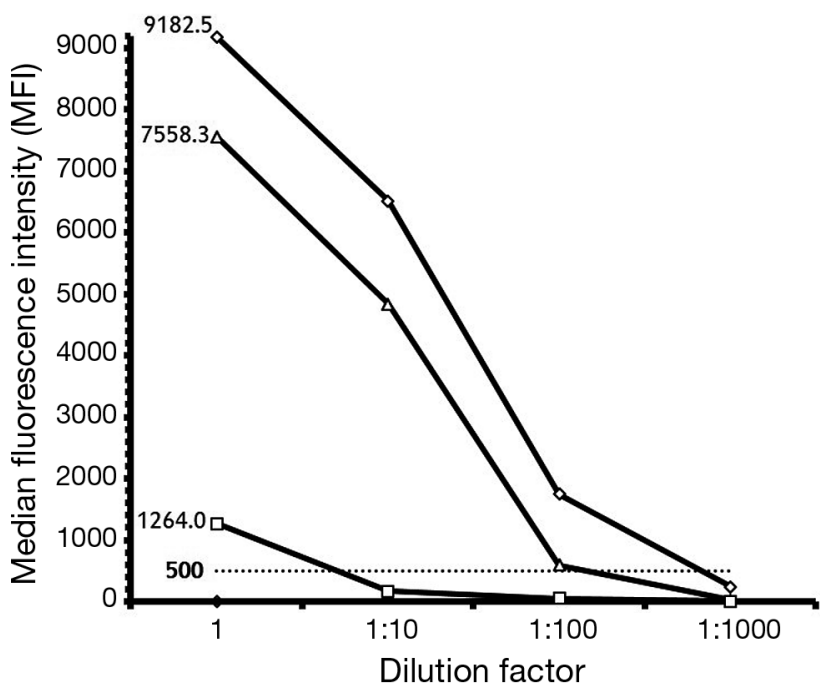

Fig. 1. Median fluorescence intensity (MFI) values of serial decimal dilutions from 3 serum samples taken from vaccinated bottlenose dolphins

(48 200 neutrophils $\mu^{-1}$ ), based on reported normal ranges in healthy animals of 5600-12 400 leukocytes $\mu \mathrm{l}^{-1}$ and 2540-6140 neutrophils $\mu^{-1}$ (Bossart et al. 2001). The test for CeMV was negative. Histopathological analysis revealed erysipelas-like lesions consisting of multisystemic intravascular bacteria and mild suppurative inflammatory reaction in affected tissues. Based on external examination, bacteriology and histopathology findings, a final diagnosis of acute E. rhusiopathiae septicemia was made (Melero et al. 2011). E. rhusiopathiae infection can progress rapidly in immunocompromised individuals 
Table 2. Median fluorescence intensity (MFI) of samples from 15 free-ranging cetaceans stranded alive in the Mediterranean Sea between 2006 and 2014. Free-ranging Risso's dolphin Grampus griseus with a positive MFI value shown in bold (see 'Results and Discussion' section). The Tursiops truncatus specimen died from acute Erysipelothrix rhusiopathiae septicemia

\begin{tabular}{|lcr|}
\hline Individual ID & Tissue & MFI \\
\hline Tursiops truncatus & & \\
Tt14.07.2010 & Serum & -110.75 \\
& $\begin{array}{c}\text { Pericardial fluid } \\
\text { Milk }\end{array}$ & -113.75 \\
Grampus griseus & & -107.50 \\
Gg25.04.2006 & Serum & \\
Gg11.12.2011 & Serum & -58.50 \\
Gg29.03.2013 & Serum & -57.50 \\
Stenella coeruleoalba & & \\
Sc09.07.2007 neonate & Serum & -21.25 \\
Sc09.07.2007 adult & Serum & -11.75 \\
Sc15.07.2007 & Serum & -13.25 \\
Sc12.07.2007 & Serum & -12.25 \\
Sc18.07.2007 & Serum & -14.25 \\
Sc25.08.2007 & Serum & -19.75 \\
Sc25.03.2011 & Serum & -118.50 \\
Sc04.12.2012 & Serum & -133.25 \\
Sc13.02.2013 & Serum & 180.75 \\
Sc12.05.2013 & Serum & -49.00 \\
Sc09.02.2014 & Serum & -107.00 \\
\hline
\end{tabular}

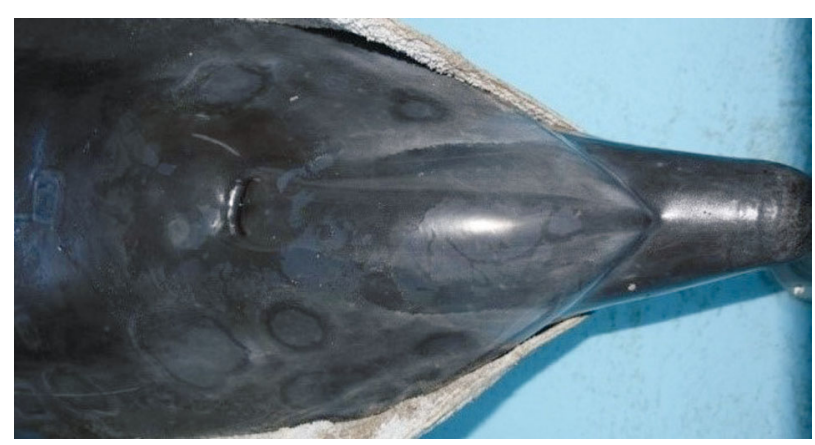

Fig. 2. Rhomboid-shaped skin lesions observed during an external examination of a bottlenose dolphin Tursiops truncatus stranded alive in the Mediterranean Sea

from other species (Sawada et al. 1987, Foster et al. 2012, Sinclair et al. 2013). In the present adult female dolphin, however, immunosuppression seems unlikely based on the marked neutrophilia and negative CeMV diagnosis. Therefore, we hypothesize that one explanation for the absence of anti-E. rhusiopathiae antibodies could be the possibility that the septicemia progressed too quickly to allow the animal to mount a specific antibody response.

From the 15 free ranging cetaceans evaluated for the presence of anti-E. rhusiopathiae antibodies, only
1 of the 3 Risso's dolphins was identified as seropositive according to Spa-based FMIA (MFI $=1649.75$ ) (Table 2). This MFI value was markedly higher than the MFI values obtained for the other 2 free ranging Risso's dolphins evaluated in this study (Table 2). Although this animal belongs to a different species than the vaccinated cetaceans, the MFI obtained was similar to the values obtained for the vaccinated animals evaluated at 6 mo after immunization (when $30 \%$ of vaccinated animals showed lower MFI value than the Risso's dolphin) and at 12 mo after immunization (when $60 \%$ of vaccinated animals showed lower MFI value) (Table 1). Detailed comparison of the MFI value from the free-ranging Risso's dolphin with the mean MFI values from vaccinated dolphins at each time point after immunization shows that the value for the free-ranging animal fell between those for the vaccinated dolphins at 12 mo (mean MFI = 1891.86) and at 13 mo (mean MFI = 161.88) (Fig. 3).

The seropositive free-ranging Risso's dolphin that was stranded alive in December 2011 was an adult male. External examination of the stranded dolphin revealed poor body condition, a moderate amount of external parasites, and the presence of secretion and parasites in the pterygoid sinuses and the tympanic area, which may have made echolocation difficult. Thus, the ability of the animal to feed effectively may have been compromised, potentially leading to it becoming weak. Nevertheless, the final cause of stranding remains unclear. This animal tested negative for $\mathrm{CeMV}$, and histopathological analysis revealed no microscopic lesions compatible with E. rhusiopathiae infection in any tissues examined. Bacteriology analysis revealed negative results for E. rhusiopathiae, Salmonella spp. and Campylobacter spp. in this animal, as well as in the other freeranging cetaceans evaluated.

Stenotrophomonas maltophilia was isolated from the cardiac valves of the seropositive Risso's dolphin. Streptococcus sp. and Staphylococcus sp. were isolated from mouth swabs of many of the free-ranging cetaceans, and bacterial species Clostridium perfringens, E. coli, and Enterobacter aerogenes were frequently isolated from intestinal samples.

The MFI value obtained from the wild Risso's dolphin (1649.75), when compared to the values obtained during several months post vaccination in 10 vaccinated bottlenose dolphins, may suggest that the Risso's dolphin was previously in contact with $E$. rhusiopathiae, though, based on the bacteriology and histopathology results, it seemed unlikely that the animal was undergoing the disease at the time of death. 


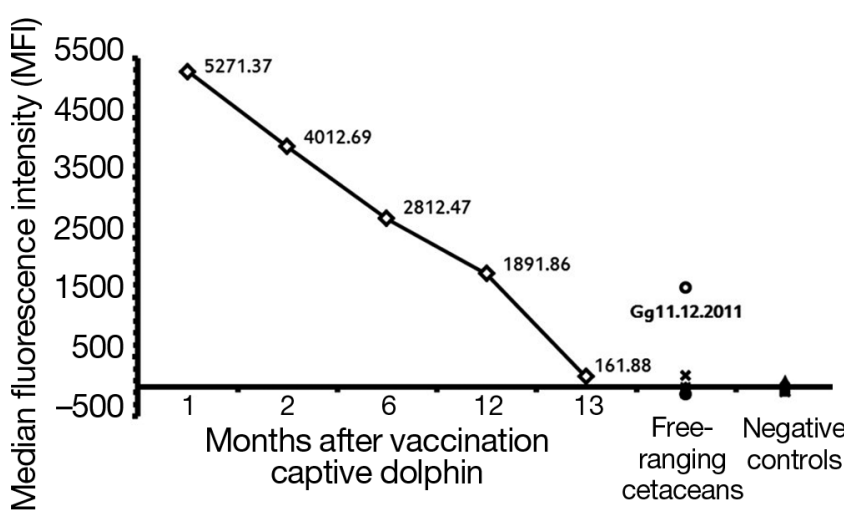

Fig. 3. Average of median fluorescence intensity (MFI) values of serum samples taken from vaccinated bottlenose dolphins at different times after annual revaccination $(n=10)$, free-ranging cetaceans stranded alive in the Mediterranean Sea between 2006 and $2014(\mathrm{n}=15)$, and unvaccinated bottlenose dolphins (negative controls) $(n=20)$. MFI value of 1649.75 from 1 free-ranging Risso's dolphin is labeled with its individual identifier (Gg11.12.2011)

\section{CONCLUSIONS}

The present study validates a fluorescent microbead-based immunoassay, based on E. rhusiopathiae Spa protein, to detect antibodies against the pathogen in cetaceans. The assay was validated in serum samples from vaccinated bottlenose dolphins, and then used successfully to assay samples from 3 species of free-ranging cetaceans: striped dolphin, Risso's dolphin and bottlenose dolphin.

FMIA can be a useful tool for vaccine efficiency assessment. This technique may be particularly valuable for studying diseases in cetaceans not only because it can be applied to free-ranging animals, but also because it allows for multiplexing. Up to 100 analytes in the same sample can be simultaneously determined, reducing the time, costs and sample volume required. This makes FMIA well suited to studies of wild dolphins, where sample collection is difficult, specimens have a great value, and where rapid, reliable diagnosis is essential.

Acknowledgements. The authors thank the entire Dolphinarium Team at the Oceanografic of the Ciudad de las Artes y las Ciencias (Valencia) for their exceptional work. This study was supported by a collaborative agreement involving the Conselleria d' Infraestructures, Territori i Medi Ambient of the Valencian Community, the Oceanographic, the VISAVET Center of Complutense University of Madrid, and the Pfizer Foundation. M.M. is the recipient of a predoctoral fellowship from the $\mathrm{PhD}$ student grant program of Complutense University of Madrid. C.R.G. is the recipient of a predoctoral fellowship from the FPU program of the Spanish Ministry of Education.

\section{LITERATURE CITED}

Anderson S, Wakeley P, Wibberley G, Webster K, Sawyer J (2011) Development and evaluation of a Luminex multiplex serology assay to detect antibodies to bovine herpes virus 1, parainfluenza 3 virus, bovine viral diarrhoea virus, and bovine respiratory syncytial virus, with comparison to existing ELISA detection methods. J Immunol Methods 366:79-88

> Bernal-Guadarrama MJ, García-Parraga D, FernándezGallardo N, Zamora-Padrón R and others (2014) Development of an indirect immunofluorescence technique for the evaluation of generated antibody titers against Erysipelothrix rhusiopathiae in captive bottlenose dolphins (Tursiops truncatus). Arch Microbiol 196:785-790

Bossart GD, Eimstad E (1988) Erysipelothrix vesicular glositis in a killer whale (Orcinus orca). J Zoo Anim Med 19: 42-47

Bossart GD, Reidarson TH, Dierauf LA, Duffield DA (2001) Clinical pathology. In: Dierauf LA, Gulland FMD (eds) Handbook of marine mammal medicine: health, disease and rehabilitation, 2nd edn. CRC Press, Boca Raton, FL, p 393-420

Brooke CJ, Riley TV (1999) Erysipelothrix rhusiopathiae: bacteriology, epidemiology and clinical manifestations of an occupational pathogen. J Med Microbiol 48:789-799

$>$ Buck JD, Spotte S (1986) Microbiology of captive whitebeaked dolphins, Lagenorhynchus albirostris, with comments on epizootics. Zoo Biol 5:321-329

Chastel C, Masure O, Balouet G, Laban P, Lucas A (1975) The student, the cetacean and swine-fever. A minor epidemic after dissection of a globicephale. Nouv Presse Med 4:1803-1805

Christopher-Hennings J, Araujo KP, Souza CJ, Fang Y and others (2013) Opportunities for bead-based multiplex assays in veterinary diagnostic laboratories. J Vet Diagn Invest 25:671-691

> Clavijo A, Hole K, Li M, Collignon B (2006) Simultaneous detection of antibodies to foot-and-mouth disease nonstructural proteins $3 \mathrm{ABC}, 3 \mathrm{D}, 3 \mathrm{~A}$ and $3 \mathrm{~B}$ by a multiplexed Luminex assay to differentiate infected from vaccinated cattle. Vaccine 24:1693-1704

> Domingo M, Visa J, Pumarola M, Marco AJ, Ferrer L, Rabanal R, Kennedy S (1992) Pathologic and immunocytochemical studies of morbillivirus infection in striped dolphins (Stenella coeruleoalba). Vet Pathol 29:1-10

Dunn JL, Buck JD, Robeck TR (2001) Bacterial diseases of cetaceans and pinnipeds. In: Dierauf LA, Gulland FMD (eds) Handbook of marine mammal medicine: health, disease and rehabilitation, 2nd edn. CRC Press, Boca Raton, FL, p 312-319

Elshal MF, McCoy JP (2006) Multiplex bead array assays: performance evaluation and comparison of sensitivity to ELISA. Methods 38:317-323

> Foster JD, Hartmann FA, Moriello KA (2012) A case of apparent canine erysipeloid associated with Erysipelothrix rhusiopathiae bacteraemia. Vet Dermatol 23:528-530

> Geraci JR, Sauer RM, Medway W (1966) Erysipelas in dolphins. Am J Vet Res 27:597-606

> Giménez-Lirola LG, Xiao CT, Halbur PG, Opriessnig T (2012) Development of a novel fluorescent microbeadbased immunoassay and comparison with three enzymelinked immunoassays for detection of anti-Erysipelothrix spp. IgG antibodies in pigs with known and unknown exposure. J Microbiol Methods 91:73-79 
Gorman R, Adley CC (2004) An evaluation of five preservation techniques and conventional freezing temperatures of $-20^{\circ} \mathrm{C}$ and $-85^{\circ} \mathrm{C}$ for long-term preservation of Campylobacter jejuni. Lett Appl Microbiol 38:306-310

> Hassanein R, Sawada T, Kataoka Y, Itoh K, Suzuki Y (2001) Serovars of Erysipelothrix species isolated from the tonsils of healthy cattle in Japan. Vet Microbiol 82:97-100

> Heaney J, Barrett T, Cosby SL (2002) Inhibition of in vitro leukocyte proliferation by morbilliviruses. J Virol 76: 3579-3584

Higgins R (2000) Bacteria and fungi of marine mammals: a review. Can Vet J 41:105-116

- Imada Y, Mori Y, Daizoh M, Kudoh K, Sakano T (2003) Enzyme-linked immunosorbent assay employing a recombinant antigen for detection of protective antibody against swine erysipelas. J Clin Microbiol 41:5015-5021

Ingebritson AL, Roth JA, Hauer PJ (2010) Erysipelothrix rhusiopathiae: association of Spa-type with serotype and role in protective immunity. Vaccine 28:2490-2496

ISO (International Organization for Standardization) (2006a) ISO 10272-1:2006. Microbiology of food and animal feeding stuffs - Horizontal method for the detection and enumeration of Campylobacter spp.-Part 1: Detection method. ISO, Geneva

ISO (International Organization for Standardization) (2006b) ISO/TS 10272-2:2006. Microbiology of food and animal feeding stuffs - Horizontal method for the detection and enumeration of Campylobacter spp.-Part 2: Colony count technique. ISO, Geneva

ISO (International Organization for Standardization) (2007) ISO 6579:2002/Amd 1:2007. Microbiology of food and animal feeding stuffs - Horizontal method for the detection of Salmonella spp. Annex D: Detection of Salmonella spp. in animal faeces and in environmental samples from the primary production stage. ISO, Geneva

Kinsel MJ, Boehm JR, Harris B, Murnane RD (1997) Fatal Erysipelothrix rhusiopathiae septicaemia in a captive Pacific white-sided dolphin. J Zoo Wildl Med 28:494-497

Kuiken T, García-Hartmann M (1991) Dissection techniques and tissue sampling. Proc 1st ECS Workshop on Cetacean Pathology: dissection techniques and tissue sampling, Leiden, Netherlands, 13-14 September 1991. European Cetacean Society Newsletter Spec Issue 17, European Cetacean Society, p 1-39

> Lacave G, Cox E, Hermans J, Devriese L, Goddeeris BM (2001) Induction of cross-protection in mice against dolphin Erysipelothrix rhusiopathiae isolates with a swine commercial vaccine. Vet Microbiol 80:247-253

Lauckner G (1985) Diseases of Mammalia: Pinnipedia. In: Kinne O (ed) Diseases of marine animals, Vol 4, Part II. Biologische Anstalt Helgoland, Hamburg, p 695-707

Medway W, Schryver HF (1973) Respiratory problems in captive small cetaceans. J Am Vet Med Assoc 163: 571-573

Melero M, Rubio-Guerri C, Crespo JL, Arbelo M and others (2011) First case of erysipelas in a free-ranging bottlenose dolphin (Tursiops truncatus) stranded in the Mediterranean Sea. Dis Aquat Org 97:167-170

Opriessnig T, Wood RL (2012) Erysipelas. In: Zimmerman JJ, Karriker LA, Ramirez A, Schwartz KJ, Stevenson GW (ed) Diseases of swine, 10th edn. Wiley-Blackwell, Oxford, p 750-759

Opriessnig T, Shen HG, Bender JS, Boehm JR, Halbur PG (2013) Erysipelothrix rhusiopathiae isolates recovered from fish, a harbour seal (Phoca vitulina) and the marine environment are capable of inducing characteristic cutaneous lesions in pigs. J Comp Pathol 148:365-372

> Rubio-Guerri C, Melero M, Rivera-Arroyo B, Bellière EN and others (2013) Simultaneous diagnosis of Cetacean morbillivirus infection in dolphins stranded in the Spanish Mediterranean sea in 2011 using a novel Universal Probe Library (UPL) RT-PCR assay. Vet Microbiol 165:109-114

> Sánchez-Matamoros A, Beck C, Kukielka D, Lecollinet S and others (2015) Development of a microsphere-based immunoassay for serological detection of African horse sickness virus and comparison with other diagnostic techniques. Transbound Emerg Dis (in press) doi: 10.1111/tbed.12340

Sawada T, Tamura Y, Takahashi T (1987) Effect of cyclophosphamide and carrageenan on resistance of mice to Erysipelothrix rhusiopathiae. Vet Microbiol 15:341-346 Schulman FY, Lipscomb TP, Moffett D, Krafft AE and others (1997) Histologic, immunohistochemical, and polymerase chain reaction studies of bottlenose dolphins from the 1987-1988 United States Atlantic Coast Epizootic. Vet Pathol 34:288-295

> Seibold HR, Neal JE (1956) Erysipelothrix septicaemia in the porpoise. J Am Vet Med Assoc 128:537-539

Sinclair M, Hawkins A, Testro A (2013) Something fishy: an unusual Erysipelothrix rhusiopathiae infection in an immunocompromised individual. BMJ Case Rep. doi: 10.1136/bcr-2013-008873

Sitt T, Bowen L, Blanchard MT, Gershwin LJ and others (2010) Cellular immune responses in cetaceans immunized with a porcine erysipelas vaccine. Vet Immunol Immunopathol 137:181-189

> Staros JV, Wright RW, Swingle DM (1986) Enhancement by N-hydroxysulfosuccinimide of water-soluble carbodiimide-mediated coupling reactions. Anal Biochem 156: 220-222

Suer LD, Vedros NA (1988) Erysipelothrix rhusiopathiae. I. Isolation and characterization from pinnipeds and bite/ abrasion wounds in humans. Dis Aquat Org 5:1-5

> Sweeney JC, Ridgway SH (1975) Common diseases of small cetaceans. J Am Vet Med Assoc 167:533-540

> Thurman GD, Downes SJ, Fothergill MB, Goodwin NM, Hegarty MM (1983) Diagnosis and successful treatment of subacute erysipelas in a captive dolphin. J S Afr Vet Assoc 54:193-200

To H, Nagai S (2007) Genetic and antigenic diversity of the surface protective antigen proteins of Erysipelothrix rhusiopathiae. Clin Vaccine Immunol 14:813-820

van Gageldonk PG, van Schaijk FG, van der Klis FR, Berbers GA (2008) Development and validation of a multiplex immunoassay for the simultaneous determination of serum antibodies to Bordetella pertussis, diphtheria and tetanus. J Immunol Methods 335:79-89

Wagner B, Freer H, Rollins A, Erb HN, Lu Z, Grohn Y (2011) Development of a multiplex assay for the detection of antibodies to Borrelia burgdorferi in horses and its validation using Bayesian and conventional statistical methods. Vet Immunol Immunopathol 144:374-381

Wang Q, Chang BJ, Riley TV (2010) Erysipelothrix rhusiopathiae. Vet Microbiol 140:405-417

Wood RL, Shuman RD (1981) Erysipelothrix infection. In: Davis JL, Karstad LH, Trainer DO (eds) Infectious diseases of wild mammals, 2nd edn. Iowa State University Press, Ames, IA, p 297-305 